\title{
Impact of CXCR4 and CXCR7 knockout by CRISPR/Cas9 on the function of triple-negative breast cancer cells
}

Meng Yang'
Chen Zeng'
Peiting Li'
Liyuan Qian'
Boni Ding'
Lihua Huang'
Gang Li'
Han Jiang'
Ni Gong
Wei Wu'
'Department of Breast Thyroid
Surgery, The Third Xiangya Hospital of
Central South University, Changsha,
Hunan 4I00I3, People's Republic of
China; ${ }^{2}$ Central Laboratory, The Third
Xiangya Hospital of Central South
University, Changsha, Hunan 4 I00I3,
People's Republic of China;
${ }^{3}$ Department of Gastrointestinal
Surgery, The Third Xiangya Hospital of
Central South University, Changsha,
Hunan Province, People's Republic
of China

Correspondence: Wei Wu

Department of Breast of Thyroid Surgery, The Third Xiangya Hospital of Central South University, Tongzi Po Road, No 172, Changsha, Hunan 410000, People's Republic of China Tel +86 73I 886I 8833 Email1103108245@qq.com

Ni Gong

Department of Gastrointestinal Surgery, The Third Xiangya Hospital of Central South University, Tongzi Po Road, No 172, Changsha, Hunan 410000, People's Republic of China $\mathrm{Tel}+8673188618232$ Email 1805278220@qq.com
This article was published in the following Dove Medical Press journal: OncoTargets and Therapy

Background: Breast cancer is one of the most common malignancies threatening women's health. Triple-negative breast cancer (TNBC) is a special type of breast cancer with high invasion and metastasis. CXCL12 and its receptors CXCR4 and CXCR7 play a crucial role in the progress of breast cancer. The aim of this study was to investigate the effect of CXCR4 and CXCR7 on the function of TNBC.

Materials and methods: We used the CRISPR/Cas9 technique to carry out a single knockout of the CXCR4 or CXCR7 gene and co-knockout of CXCR4 and CXCR7 genes in the TNBC cell line (MDA-MB-231). The single knockout and co-knockout cells were screened and verified by PCR sequencing and Western blot assay, the effect of single knockout and co-knockout on the proliferation of TNBC cells was examined using the Cell Counting Kit- 8 and colony formation assays, the migration and invasion of TNBC cells were examined by the transwell and wound-healing assays, the changes in the cell cycle distribution after knockout were detected by flow cytometry, and the difference in the migration and invasion of single knockout and co-knockout induced by CXCL12 was observed by adding CXCL12 in the experimental group. Results: The single knockout of the CXCR4 or CXCR7 gene significantly reduced the cell proliferation, growth, migration, and invasion and delayed the conversion of the G1/S cycle, while the co-knockout inhibited these biological abilities more significantly. In both the knockout and control groups, the migration and invasion of CXCL12-added cells were significantly stronger than those of the non-CXCL12-added cells, and CXCL12 induced lesser migration and invasion in the CXCR4 and CXCR7 co-knockout group than in the single knockout groups. Conclusion: The knockout of the CXCR4 and CXCR7 genes affects the binding capacity and functions of CXCL12, inhibits the malignant progression of TNBC cells significantly, and may become a potential target for the treatment of TNBC.

Keywords: triple-negative breast cancer cells, TNBC, CXCR4, CXCR7, CXCL12, CRISPR/Cas9

\section{Introduction}

Breast cancer is one of the most common malignant tumors threatening women's health and is one of the most common causes of cancer death in women; ${ }^{1}$ additionally, the number of new patients per year in China has increased by $3 \%-4 \%$. ${ }^{2}$ Triple-negative breast cancer (TNBC), with negatively expressed ER, PR, and HER2, is a special type of breast cancer with high morphologic and prognostic heterogeneity. ${ }^{3}$ Its rates of invasion, distant metastasis, and mortality are higher than those of the other types of breast cancer. ${ }^{4}$ Because the effect of endocrine therapy on TNBC is unsatisfactory and no effective specific targeted therapy is available, the treatment and prognosis management of TNBC remains a clinical challenge. 
Chemokines and their receptors play important roles in the metastasis and development of tumors, ${ }^{5}$ but their specific mechanisms in affecting metastasis remain to be further clarified. CXCL12 (also known as stromal cellderived factor) is associated with the progression of many cells, especially tumor cells, and is highly expressed in many organs resulting in easy metastasis of breast cancer cells to these organs, such as the lymph node, liver, lung, and bone marrow. Moreover, its high expression in breast cancer cells is associated with invasion, metastasis, and a poor prognosis. ${ }^{6,7}$ CXCL12 interacts with its homologous receptors CXCR4 and CXCR7 to regulate cell proliferation, invasion, migration, and angiogenesis. ${ }^{89} \mathrm{CXCR} 4$ is a G-protein-coupled receptor associated with the $\mathrm{G} \alpha$ subunit, ${ }^{10}$ which has four different $\mathrm{G} \alpha$ subunits to activate different downstream signaling pathways, so that the transduction of the $\mathrm{G}$ protein-coupled receptor signal to different pathways affects the function of tumors. ${ }^{11}$ CXCR4 is expressed continuously in human breast cancer cells, and its ligand CXCL12 is preferentially expressed in the lung, liver, and lymphoma. ${ }^{12}$ Additionally, CXCL12 demonstrates a chemotactic effect on tumor cells expressing CXCR4, which can cause specific metastasis of tumors to form metastatic foci. ${ }^{13,14}$ Therefore, the CXCL12/CXCR4 signaling axis is thought to play a key role in the development of breast cancer metastasis. ${ }^{15}$ CXCR7, another receptor of CXCL12, plays a more complex role in the progression of breast cancer and is highly expressed in the vascular system of primary tumors. ${ }^{16,17} \mathrm{CXCR} 7$ activates tumor cells to secrete vascular endothelial growth factor to promote tumor angiogenesis and enhance the adhesion of cancer cells to fibrin and endothelial cells, thus promoting the metastasis of cancer cells. ${ }^{17-19}$ Studies have shown that CXCR7 is highly expressed in breast cancer tissue, promoting the growth, proliferation, invasion, and metastasis of tumor cells, and its high expression is closely related to the metastasis of tumors to the lung. ${ }^{19}$ Additionally, CXCR7 can enhance cell adhesion, invasion, and angiogenesis in vitro, and it can promote tumor growth in vivo. ${ }^{20,21}$ Other studies have shown that CXCR7 can promote the proliferation and migration of in vitro-cultured tumor cells toward CXCL12. ${ }^{22-25}$ However, the specific mechanism of the CXCL12/CXCR7 axis in the invasion and metastasis of breast cancer remains unclear.

Compared with other types of breast cancer, TNBC has a higher distant recurrence, visceral metastasis, and death rate. ${ }^{26}$ Our previous studies have shown that the overexpression of CXCL12 and its receptors CXCR4 and CXCR7 in breast cancer is associated with a poor prognosis and metastasis, ${ }^{27}$ and their expression levels are higher in TNBC than in other types of breast cancer. ${ }^{28}$ Thus, whether the poor prognosis of TNBC patients is related to the high expression of these three factors has not been reported, and the exact mechanism underlying how the three factors affect the metastasis and development of TNBC is unknown. In this study, the CRISPR/Cas9 technique was used for the single knockout and co-knockout of the CXCR4 or/and CXCR7 target gene in TNBC cells (MDA-MB-231), to explore the change in the biological functions (such as proliferation, migration, and invasion) of TNBC cells, and to investigate the biological effects of CXCL12 on control and knockout cells, all of which can further elucidate the roles of chemokine CXCL12 and its receptors CXCR4 and CXCR7 in the metastasis process of TNBC and provide possible effective therapeutic targets for the gene therapy of TNBC.

\section{Materials and methods}

Establishment of stable cell lines of the single knockout and co-knockout of the CXCR4 and/or CXCR7 gene using the CRISPR/Cas9 double-vector lentivirus

\section{Cell culture}

The TNBC cell line MDA-MB-231 (Genechem Company, Shanghai, China) was cultured in L-15 medium (Keygen Biotech, Nanjing, China), and supplemented with $10 \%$ FBS (BioInd, Kibbutz Beit Haemek, Israel) in a humidified atmosphere at $37^{\circ} \mathrm{C}$ with $5 \% \mathrm{CO}_{2}$ (kept in the Central Laboratory of Third Xiangya Hospital of Central South University).

\section{Design of lentivirus}

Double-vector lentivirus was designed and manufactured by Genechem Company, custom SgRNAs for CXCR4/ CXCR7 were designed using the website (http://zlab.bio/ guide-design-resources) with the sequence of CXCR4 (NM_003467.2) and CXCR7 (NM_020311.3). The knockout target sequence of CXCR4 is located in exon 2, while the knockout target sequence of CXCR7 is located in exon 1 (Figure 1A and B).

\section{Vector lentivirus transfection}

We used the Cas9 lentivirus designed and produced by Genechem Company to transfect the MDA-MB-231 cells. The mixed clones with stable expression of Cas9 were screened using puromycin resistance and then SgRNA lentivirus for CXCR4 (with green fluorescence) or CXCR7 (with red fluorescence) gene single knockout was added (virus volume $(10 \mu \mathrm{L})=$ multiplicity of infection $(10) \times$ Cell number 

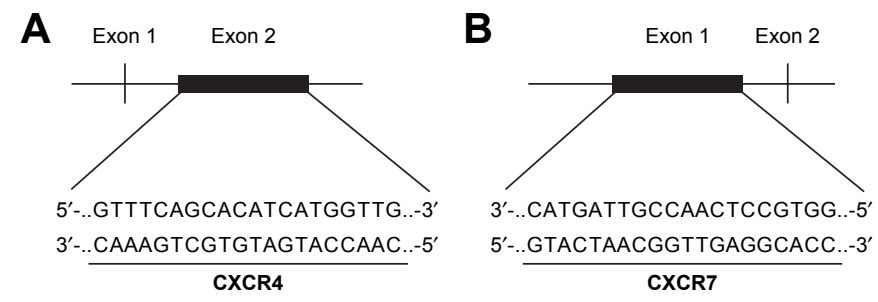

C
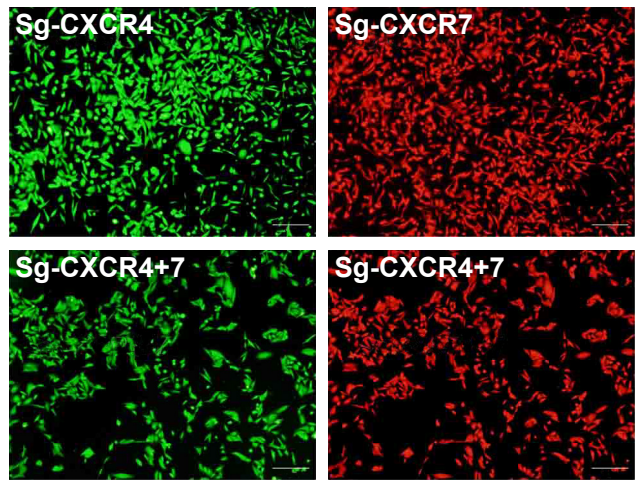

G

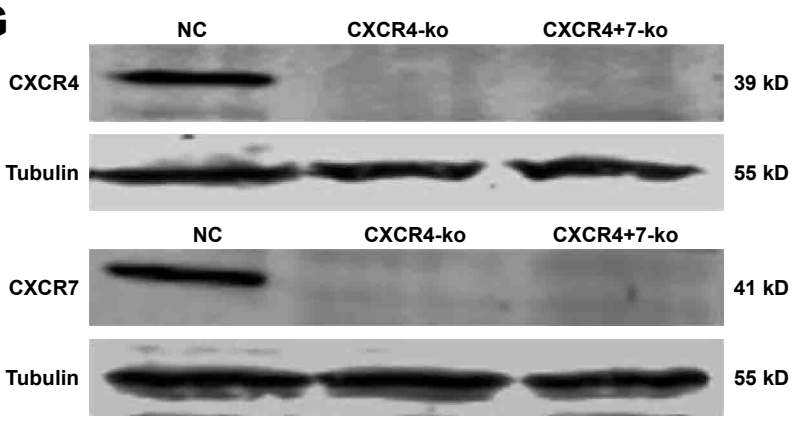

D

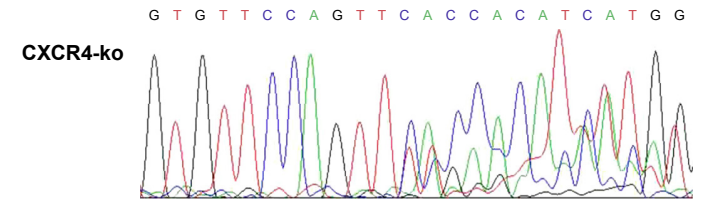

ORIG: GTGTTCCAGTTTCAGCACATCATGGTTGG

READ: GTGTTCCAGTTCACCACATCATGGGTGGG

UNRE: GTGTTCCAGTTTTCAAATATCATTGTTCG

FRST: GTGTTCCAGT-TCAGCACATCATGGTTG (1 nt del)

SCND: GTGTTCCAGT--------ATCATGGTTG (8 nt del)

\section{E}

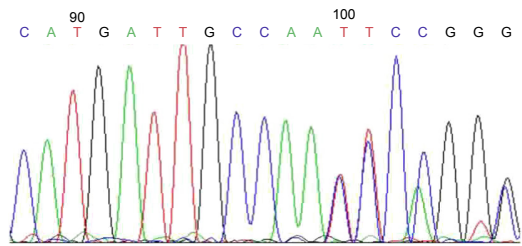

CXCR7-ko

ORIG: TGATTGCCAACTCCGTGGTGGTCTGGGTGAATATCCAGGC

READ: TGATTGCCAATTCCGGGCGAAAATCCAGGCTATGACCACC

UNRE: TGATTGCCAACCTAGGCTAAGTCCACAGGCCAAAACACGA

FRST: TGATTGCCAAC-----------TCTGGGTGAATATCCAGGC (10 nt del)

SCND: TGATTGCCAA-_-_-_-_-_-__-_-_-_-_-_TCCAGGC (23 nt del)

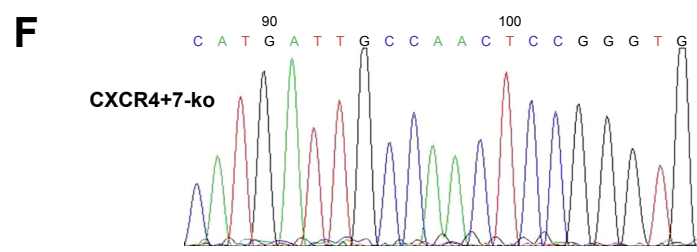

ORIG: TGATTGCCAACTCCGTGGTGGTC

READ: TGATTGCCAACTCCGGGTGGTC

MUTA: TGATTGCCAACTCCG-GGTGGTC (1 nt del)

Figure I Design of lentivirus, transfection, screening, and PCR sequencing of single-knockout (Sg-CXCR4 and Sg-CXCR7) and co-knockout (Sg-CXCR4+7) cell clones and verification of the protein expression level by Western blotting. (A and B) The knockout target sequence of CXCR4 is located in its exon 2, while the knockout target sequence of CXCR7 is located in exon I. (C) In MDA-MB-23I cells, after transfection with Cas9/SgRNA lentivirus, the transfection efficiency was significant. (D-F) The PCR products of monoclonal cells after the knockout of CXCR4 and CXCR7 target genes were sequenced. (G) Western blotting was used to detect the expression of CXCR4 and CXCR7 in monoclonal cells.

Abbreviations: NC, negative control; ORIG, original; UNRE, unread; FRST, first; SCND, second; nt, nucleotide; MUTA, mutation.

$\left(1 \times 10^{9}\right) /$ Virus titer $\left.\left(1 \times 10^{8} \mathrm{TU} / \mathrm{mL}\right)\right)$. The SgRNA sequences were as follows: CXCR4, 5' GTTTCAGCACATCATGGTTG 3'; CXCR7, 5' CATGATTGCCAACTCCGTGG 3'. The cells expressing Sg-CXCR4 and Sg-CXCR7 stably were selected by a monoclonal technique. Briefly, the technique involves digestion and dilution of transfected cells $\left(1 \times 10^{4} / \mathrm{mL}\right)$. Continue to dilute the cell fluid concentration to $10 / \mathrm{mL}$, add $100 \mu \mathrm{L}$ to each hole in 96 holes, equivalent to one cell per hole. Then the cells are cultured at $37^{\circ} \mathrm{C}$ in a humidified incubator with 5\% $\mathrm{CO}_{2}$ for 10-14 days until the cell mass formation occurs. Next, the cells were subjected to trypsin digestion, transfered to a 24-well plate, and continued to be cultured for $7-10$ days. Until cell convergence reached $70 \%-80 \%$ digesting to six-hole plate, when about $80 \%$ confluence is to be cultured. The final step includes trypsin digestion and extraction of DNA and protein for further validation.
Establishment of co-knockout cell lines

For co-knockout, the CXCR7 SgRNA lentivirus (with red fluorescence) was added to the cells with stable single knockout of the CXCR4 gene, and then the cell lines with stable co-knockout of CXCR4 and CXCR7 (CXCR4+7) were selected by using a monoclonal technique. A virus with $\mathrm{Sg}-\mathrm{NC}$ as the scramble sequence (NC) was used to obtain the negative control.

\section{Monoclonal screening and identification of cells with the single knockout and co-knockout of the CXCR4 and/or CXCR7 gene PCR}

The monoclonal cell DNA was extracted and subjected to PCR amplification, the PCR product was sequenced, and the knockout of the CXCR4 and/or CXCR7 gene was verified 
at the DNA level. Monoclonal cell DNA was extracted using the DNA extraction kit (Omega, Norcross, GA, USA) and primer sequences were designed by Genechem Company: CXCR4-F: 5'-CTGACTATTCCCGACTTCATCTT TG-3'; CXCR4-R: 5'-CCAATGTAGTAAGGCAGCCA ACAG-3'; CXCR7-F: 5'-CATGCAACAGCAGCGACTGC ATCG-3'; CXCR7-R: 5'-CAGCGATAATGGAGAA GGGAACG-3'. PCR amplification was carried out according to the following reaction conditions: $94^{\circ} \mathrm{C}$ for 5 minutes, followed by $94^{\circ} \mathrm{C}$ for 30 seconds, $55^{\circ} \mathrm{C}$ for 30 seconds, and $72^{\circ} \mathrm{C}$ for 40 seconds for 34 cycles and then $72^{\circ} \mathrm{C}$ for 5 minutes. The PCR products were subjected to agarose gel electrophoresis to detect the target band and then were sent to Tsingke Biotech Company (Changsha, China) for sequencing.

\section{Western blotting}

Western blotting was used to verify whether the CXCR4 and/or CXCR7 protein was expressed in the monoclonal cells. Total protein was extracted from the cell lines using RIPA buffer (Keygen Biotech) following the manufacturer's protocol. The total protein lysates were heated with SDSPAGE sample loading buffer (Biosharp, Seoul, Korea) and then kept at $-80^{\circ} \mathrm{C}$ until use. The denatured protein extracts $(40 \mu \mathrm{g})$ were then subjected to $10 \%$ SDS-PAGE and were transferred to polyvinylidene difluoride (PVDF) membranes (EMD Millipore, Billerica, MA, USA) electrophoretically. After blockade with 5\% degreased milk and washing with PBS for three times (each for 10 minutes), the membranes were incubated overnight at $4{ }^{\circ} \mathrm{C}$ with primary antibodies: mouse anti-CXCR4 (1:500; Proteintech, Rosemont, IL, USA), rabbit anti-CXCR7 (1:1,000; Abcam, Cambridge, UK), and mouse anti-Tubulin (1:10,000; Proteintech). The PVDF membranes were then washed with PBS for three times (each for 10 minutes) and incubated with infrared fluorescence-labeled secondary antibodies for 1 hour. Finally, the membranes were washed with PBS three times (each for 10 minutes). The protein band signals were detected and analyzed for the signal intensity using the Odyssey Clx system (Licor Company, Lincoln, NE, USA).

\section{Colony formation assay}

NC, CXCR4-ko, CXCR7-ko, and CXCR4+7-ko cells (each $5 \times 10^{2}$ cells) were counted and seeded into six-well plates and then cultured at $37^{\circ} \mathrm{C}$ in a humidified incubator with $5 \%$ $\mathrm{CO}_{2}$ for 10-12 days until the colonies were visible. The cell colonies were fixed with methanol for 30 minutes and then were stained with $0.1 \%$ crystal violet for 30 minutes and photographed, followed by counting of the number of colonies.

\section{Cell proliferation assay}

L15 medium $(100 \mu \mathrm{L})$ containing quantified 4,000 control (NC) or knockout (CXCR4-ko, CXCR7-ko, and CXCR4+7ko) cells was added to 96-well plates and cultured for 24, 48, 72 , and 96 hours after the cells were seeded. Next, $10 \mu \mathrm{L}$ of Cell Counting Kit (CCK-8) solution (Dojindo, Kumamoto, Japan) was added to each well at each time point. The cells were cultured with CCK- 8 for 3 hours, and then the absorbance at $450 \mathrm{~nm}$ was measured using the BioTek ELX800 system (BioTek, Winooski, VT, USA).

\section{Transwell assay for determining cell migration and invasion}

For the migration assay, NC, CXCR4-ko, CXCR7-ko, and CXCR4+7-ko cells $\left(2 \times 10^{5}\right.$ in $200 \mu \mathrm{L}$ of serum-free L-15 medium) were seeded into the upper chamber of 24-well transwell plates with a pore diameter of $8 \mathrm{~mm}$ (Corning Costar, Corning, NY, USA). Next, $700 \mathrm{~mL}$ of medium containing $10 \%$ FBS was added to the bottom chamber as a chemoattractant. After 24-48 hours of culture, the cells were fixed with methanol for 30 minutes, and $0.1 \%$ crystal violet staining was performed. After dye staining for 30 minutes, the cells were rinsed with PBS, and a cotton swab was used to wipe off the residual cells in the apical membrane of the ventricle. Five random fields were visualized under a whitelight microscope (Olympus, Tokyo, Japan) and counted.

For the invasion assay, the top chambers were coated with basement membrane Matrigel (BD Biosciences, San Jose, CA, USA) at $37^{\circ} \mathrm{C}$ for 30 minutes. NC, CXCR4-ko, CXCR7-ko, and CXCR4+7-ko cells $\left(3 \times 10^{5}\right)$ with serum-free medium were added to the top chambers, and the bottom chambers were filled with medium containing 10\% FBS. After 24-48 hours of culture, the cells were fixed with methanol and were stained with crystal violet solution. The remaining cells were removed from the top of the permeable membrane using a cotton swab. Next, the cells that migrated through the upper chamber were counted in five random fields under a light microscope (Olympus), and the average value of the five fields was calculated.

\section{Wound-healing assay}

NC, CXCR4-ko, CXCR7-ko, and CXCR4+7-ko cells (5×105) were inoculated into a six-well plate with parallel horizontal lines on the back. The cells were incubated in an incubator $\left(37^{\circ} \mathrm{C}, 5 \% \mathrm{CO}_{2}\right)$ overnight. After $80 \%-90 \%$ confluency was reached, the wells were rinsed with $\mathrm{PBS}$, added to serumfree medium, cultured in an incubator $\left(37^{\circ} \mathrm{C}, 5 \% \mathrm{CO}_{2}\right)$, and photographed under a light microscope at $0,12,24,36,48$, and 60 hours. The migration area and migration rate were calculated using AI software (Adobe Illustrator CC 2018). 


\section{Cell cycle assay}

The cells (NC, CXCR4-ko, CXCR7-ko, and CXCR4+7-ko) were trypsinized and fixed with $70 \%$ ethanol at $4^{\circ} \mathrm{C}$ overnight. Next, $3 \times 10^{5}$ cells were resuspended in $0.5 \mathrm{~mL}$ of PI/ RNase staining buffer (BD Biosciences). After incubation in the dark at room temperature for 15 minutes, the cells were characterized using a BD FACS Verse apparatus, and the data were analyzed using Modfit LT 3.1 software. The experiments were carried out in triplicate.

\section{Statistical analysis}

Each trial was repeated at least three times, and the data were expressed as the mean \pm SD. The data were compared between groups by independent sample $t$-test and paired sample $t$-test, and repeated measurements were made at different time points for a certain subject. The ANOVA was performed for repeated measurements. $P<0.05$ was considered to be statistically significant, and Graphpad Prism 7 was used for statistical mapping.

\section{Results}

\section{Screening and identification of CXCR4 and CXCR7 gene single knockout and co-knockout cell clones}

After adding Cas9 and SgRNA double-vector lentivirus to TNBC cells (MDA-MB-231), the rate of transfection cells was high, as seen from the green (for Sg-CXCR4) and red (for Sg-CXCR7) fluorescence, as shown in Figure 1C. After monoclonal screening, DNA from $\sim 50$ clones was extracted for PCR amplification. The PCR products in agarose gel electrophoresis showed that the target bands of Sg-CXCR4 and Sg-CXCR7 were $\sim 312$ bp and 630 bp in length, respectively, consistent with the expected design. The sequencing result of a Sg-CXCR4 clone (Figure 1D) showed that the front part was single-peaked, but the later nucleotides were double-peaked, indicating a difference between two alleles. On comparing the read and unread sequences with the original sequence, the sequences of the mutated alleles were obtained. The number of deleted nucleotides (1 and 8) was not threefold, indicating the read frame was shifted. The SgRNA recognition position was designed in the first exon; thus, the CXCR4 gene of this cell clone showed biallelic mutation, and then the CXCR4 knockout cell line was successfully constructed and termed CXCR4-ko. Likewise, the CXCR7-ko cell line was successfully constructed (Figure 1E). By adding Sg-CXCR7 to the CXCR4-ko cell line, another CXCR7 mutation was identified, whose both alleles had one nucleotide deleted (Figure 1F); thus, this cell clone showed the knockout of both CXCR4 and CXCR7 genes and was termed as CXCR4+7-ko.

\section{Verification of CXCR4 and CXCR7 gene knockout by protein detection}

Western blot results (Figure 1G) showed that, compared with NC, CXCR4-ko or CXCR7-ko cells did not express CXCR4 or CXCR7 protein, respectively; CXCR4+7-ko cells expressed neither CXCR4 protein nor CXCR7 protein. Thus, the CXCR4 and CXCR7 genes single knockout and CXCR4+7 co-knockout stable strains were successfully verified at the protein level.

\section{CXCR4 and CXCR7 gene knockout inhibits the proliferation of breast cancer cells and prolongs the cell cycle}

The numbers of colonies formed after 10-12-day culture of NC, CXCR4-ko, CXCR7-ko, and CXCR4+7-ko cells was $204.3 \pm 3.18,92.0 \pm 2.31,94.7 \pm 5.78$, and $57.67 \pm 3.18$ (mean $\pm \mathrm{SD} ; \mathrm{n}=3$ ), respectively (Figure $2 \mathrm{~A}$ and $\mathrm{B}$ ). Compared with the $\mathrm{NC}$ group, the colony formation abilities
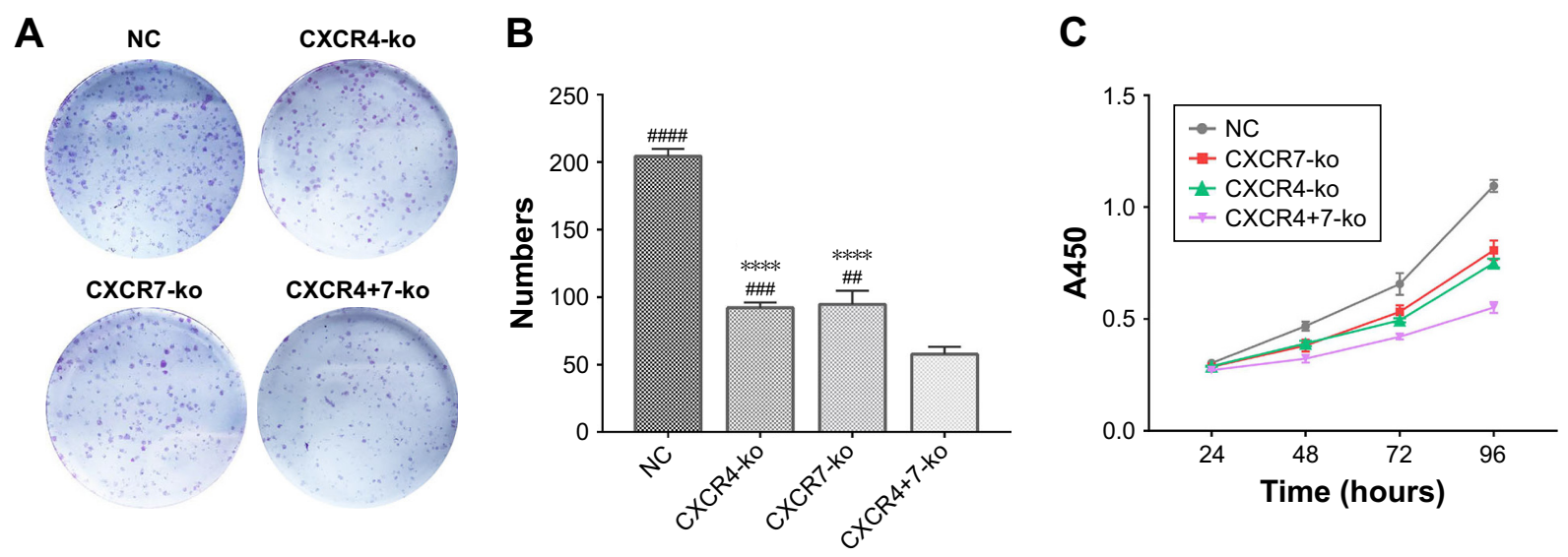

Figure 2 (Continued) 

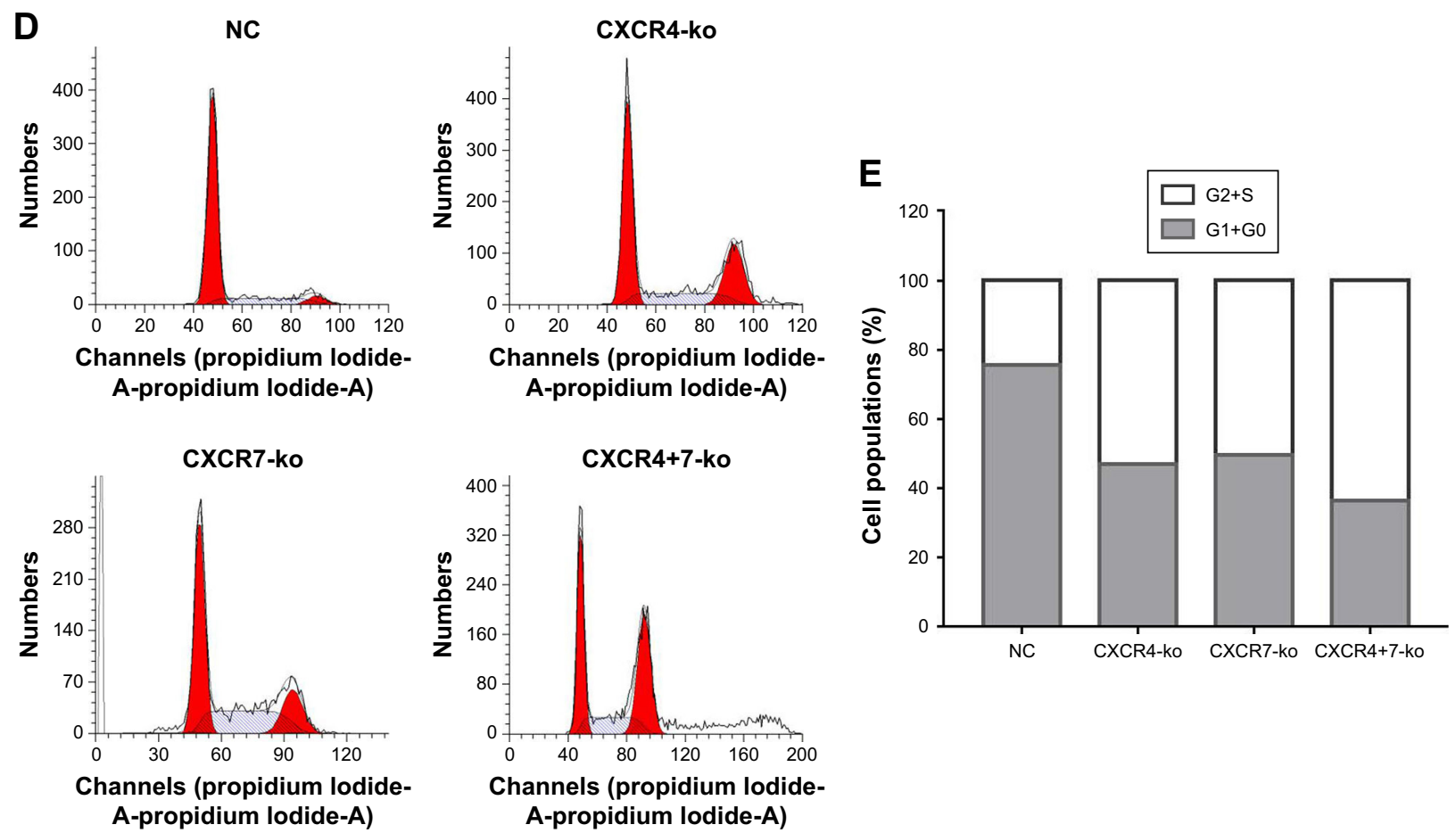

Figure 2 Knockout of target genes CXCR4 and CXCR7 can significantly inhibit cell proliferation and cell cycle changes. Colony formation (A and B) and CCK-8 (C) assays indicated that the knockout of CXCR4 and/or CXCR7 represses TNBC cell proliferation. Moreover, the co-knockout effect was more significant than that of single knockout. The representative images and quantification of flow cytometry analysis of cells after transfection is shown. Knockdown of CXCR4 and/or CXCR7 reduced the number of cells in the GI phase but promoted the number of cells in the $S$ and G2/M phases ( $\mathbf{D}$ and $\mathbf{E}$ ). The inhibition in the co-knockout group was more significant.

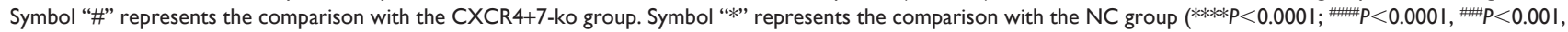
$\# P<0.0$ I). The data were analyzed in triplicate, and similar results were obtained. Representative images are illustrated.

Abbreviations: CCK, Cell Counting Kit; TNBC, triple-negative breast cancer; NC, negative control.

of CXCR4-ko, CXCR7-ko, and CXCR4+7-ko cells were decreased significantly by $55.5 \%, 53.7 \%$, and $71.8 \%$, respectively $(P<0.01)$. The colony formation ability of CXCR4+7-ko cells decreased significantly compared with that of CXCR4-ko or CXCR7-ko cells $(P<0.01)$.

The CCK-8 absorbance values after 4-day culture of NC, CXCR4-ko, CXCR7-ko, and CXCR4+7-ko cells were $1.10 \pm 0.016,0.75 \pm 0.011,0.81 \pm 0.025$, and $0.55 \pm 0.014(n=3)$, respectively, as shown in Figure 2C. Compared with the NC group, the cell proliferation rates of CXCR4-ko, CXCR7-ko, and CXCR4+7-ko cells were reduced significantly by $\sim 32 \%$, $26 \%$, and $50 \%$, respectively $(P<0.01)$. The cell proliferation ability of CXCR4+7-ko cells decreased significantly compared with that of CXCR4-ko or CXCR7-ko cells $(P<0.001)$.

Flow cytometry analysis showed the cell cycle G0+G1\% distribution of NC, CXCR4-ko, CXCR7-ko, and CXCR4+7-ko cells (Figure 2D and E). Compared with the NC group, the G0+G1\% values of CXCR4-ko, CXCR7-ko, and CXCR4+7-ko cells were reduced significantly by $\sim 32 \%, 26 \%$, and $50 \%$, respectively $(P<0.01)$. The $\mathrm{G} 0+\mathrm{G} 1 \%$ of CXCR4+7-ko cells was decreased significantly compared with that of CXCR4-ko or CXCR7-ko cells $(P<0.01)$. Thus, CXCR4 and CXCR7 knockout could delay the G1/S cycle conversion and promote the number of cells in the $\mathrm{S}$ and $\mathrm{G} 2 / \mathrm{M}$ phases.

\section{CXCR4 and CXCR7 knockout can inhibit the migration ability of TNBC cells}

Transwell assays showed that the numbers of cells migrated from the upper chamber to the bottom chamber without CXCL12 were $477 \pm 6.245,142 \pm 5.132,179.3 \pm 2.848$, and $47.33 \pm 3.18(\mathrm{n}=3)$ for the NC, CXCR4-ko, CXCR7-ko, and CXCR4+7-ko cells, respectively (Figure 3A and C). Compared with the NC group, the cell migration of CXCR4-ko, CXCR7-ko, and CXCR4+7-ko cells was inhibited by $70.2 \%$, $62.4 \%$, and $90.1 \%$, respectively, with a significant difference $(P<0.01)$. The cell migration of $\mathrm{CXCR} 4+7-$ ko cells was decreased significantly compared with that of CXCR4-ko or CXCR7-ko cells $(P<0.01)$.

When CXCL12 cells were added to the bottom chamber, the transwell assays showed that the numbers of cells that migrated from the upper chamber were $717.7 \pm 23.92$, $255.7 \pm 3.756,331.7 \pm 3.18$, and 100.3 $\pm 2.186(n=3)$ for the NC, CXCR4-ko, CXCR7-ko, and CXCR4+7-ko cells, 

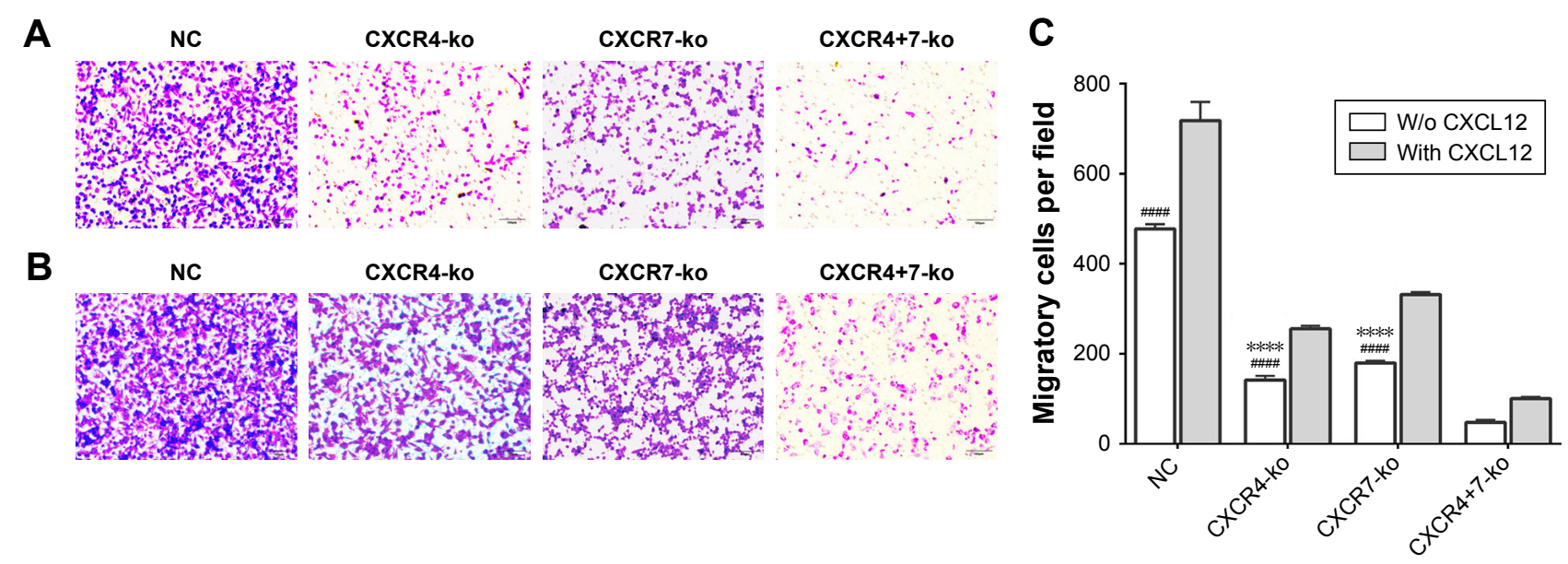

D
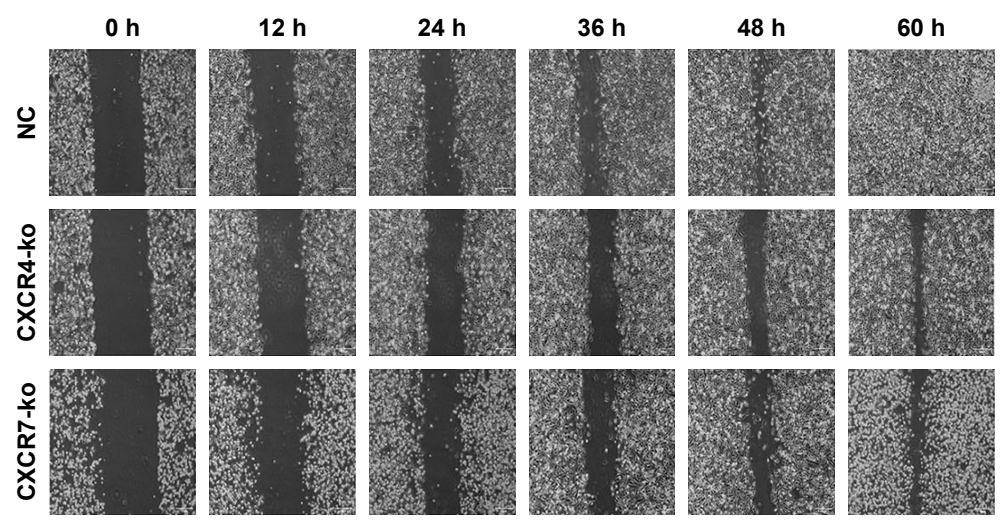

E
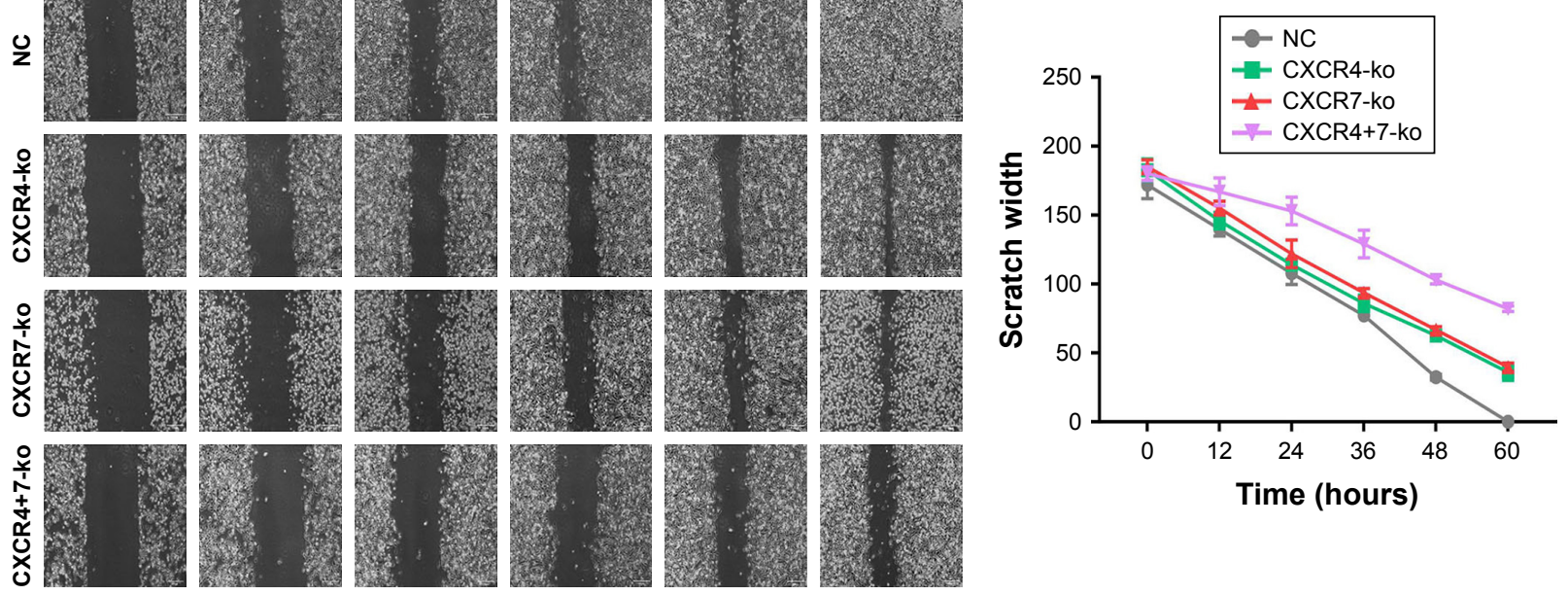

Figure 3 Knockout of the target genes CXCR4 and CXCR7 can significantly inhibit the migration ability of TNBC cells. The transwell migration assay showed that single knockout and co-knockout of CXCR4 and CXCR7 significantly inhibited the migration of TNBC cells, and co-knockout was more effective. Regardless of the control group or experimental group, the addition of chemokine CXCLI2 significantly increased the migration ability of TNBC cells (A) no added CXCLI2; (B) added CXCLI2; (C) is an analysis of the results of graphs A and B. Wound healing showed that single knockout and co-knockout of CXCR4 and CXCR7 resulted in a significant decrease in cell migration (D and E). Symbol “*”" represents the comparison with the NC group. Symbol “\#” represents the comparison with the CXCR4+7-ko group (****P<0.000I; \# $P<0.000$ I). The data were analyzed in triplicate, and similar results were obtained. Representative images are illustrated.

Abbreviations: TNBC, triple-negative breast cancer; NC, negative control; w/o, without.

respectively (Figure 3B and C). The cell migration capacity of CXCR4+7-ko cells was decreased significantly compared with that of CXCR4-ko or CXCR7-ko cells $(P<0.01)$.

The initial scratch width observed in wound healing experiments was $1.8 \mathrm{~mm}$. After 60 hours of culture, the wound width was found to be $0,0.35 \pm 0.029,0.365 \pm 0.032$, and $0.82 \pm 0.012 \mathrm{~mm}(\mathrm{n}=3)$ for NC, CXCR4-ko, CXCR7-ko, and CXCR4+7-ko cells, respectively. When compared with the NC cells, the percentage decrease of width values for CXCR4-ko, CXCR7-ko, and CXCR4+7-ko cells were 80.6\%, $79.7 \%$, and $54.4 \%$, respectively (Figure 3D and E), with a significant difference $(P<0.01)$. Compared with CXCR4-ko or CXCR7-ko cells, the migration of CXCR4+7-ko cells was inhibited significantly $(P<0.01)$.

\section{CXCR4 and CXCR7 knockout inhibit the invasion ability of TNBC cells}

The cell invasion abilities of CXCR4 and CXCR7 knockout cells were studied using the transwell assay with Matrigel in the upper chamber. The results showed that the numbers of cells that migrated from the upper chamber to the bottom chamber without CXCL12 were $392.7 \pm 13.54$, $125.7 \pm 5.812,158.3 \pm 6.936$, and $40.67 \pm 4.333(n=3)$ for the NC, CXCR4-ko, CXCR7-ko, and CXCR4+7-ko cells, respectively (Figure 4A and C). Compared with the NC group, the cell migration rates of CXCR4-ko, CXCR7-ko, and CXCR4+7-ko cells were inhibited by $68.0 \%, 59.7 \%$, and $89.6 \%$, respectively, with a significant difference $(P<0.01)$. The cell migration rate of CXCR4+7-ko cells was 

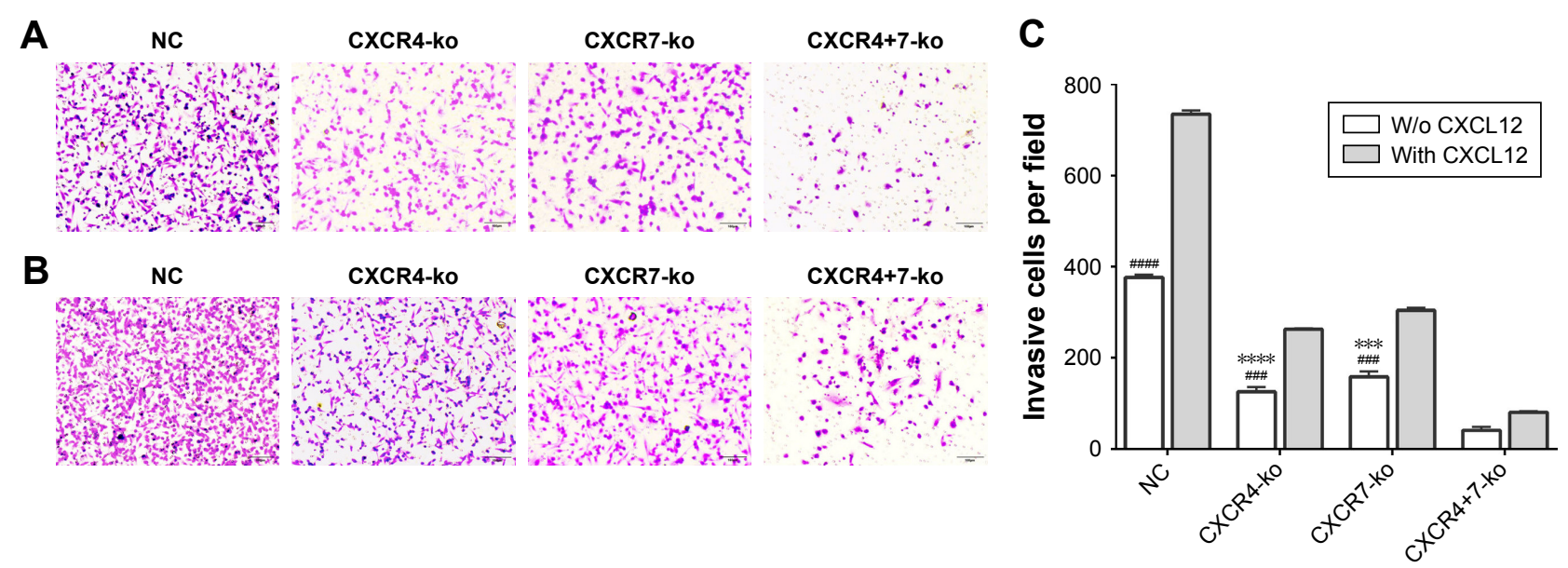

Figure 4 Knockout of the target genes CXCR4 and CXCR7 can significantly inhibit the invasion ability of TNBC cells. The transwell invasion assay showed that single knockout and co-knockout of CXCR4 and CXCR7 significantly inhibited the invasion of TNBC cells, and co-knockout was more effective. Regardless of the control group or experimental group, the addition of chemokine CXCLI2 significantly increased the migration ability of TNBC cells (A-C). Symbol "**" represents the comparison with the NC group. Symbol “\#” represents the comparison with the CXCR4+7-ko group (****P<0.000I, $* * * P<0.001$; $\# \# P<0.0001$, \#\#P<0.00I). The data were analyzed in triplicate, and similar results were obtained. Representative images are illustrated.

Abbreviations: TNBC, triple-negative breast cancer; NC, negative control, w/o, without.

decreased significantly compared with that of CXCR4-ko or CXCR7-ko cells $(P<0.01)$.

When CXCL12 cells were added to the bottom chamber, the transwell assay showed that the numbers of cell that invaded from the upper chamber were $734.7 \pm 4.702$, $262.7 \pm 1.333,304 \pm 3.215$, and $80.33 \pm 1.453(\mathrm{n}=3)$ for the $\mathrm{NC}$, CXCR4-ko, CXCR7-ko, and CXCR4+7-ko cells, respectively (Figure 4B and C). Compared with the NC group, the cell migration abilities of CXCR4-ko, CXCR7-ko, and CXCR4+7-ko cells were inhibited significantly $(P<0.01)$. The cell migration rate of CXCR4+7-ko cells was decreased significantly compared with that of CXCR4-ko or CXCR7ko cells $(P<0.01)$.

\section{Discussion}

TNBC is characterized by high metastasis and rapid progress compared with the other breast cancer subtypes. Metastasis is the main cause of death and an independent prognostic factor of breast cancer, which is a complex, ordered, multistep, nonrandom, highly organoselective pathophysiological process. ${ }^{29}$ The chemokine CXCL12 and its receptors CXCR4 and CXCR7 play an important role in tumorigenesis and metastasis; CXCR4 and CXCR7 affect the progression of TNBC through different signaling pathways; however, the specific mechanism by which they exert their influence on TNBC needs to be further elucidated. In this study, the CRISPR/Cas9 technique was used in the TNBC cell line MDA-MB-231 to establish monoclonal stable cell lines with CXCR4 and CXCR7 single knockout and co-knockout methods. The clinical data also showed that high expression levels of CXCL12 and its receptors CXCR4 and CXCR7 were associated with easy metastasis and poor prognosis of TNBC. ${ }^{27}$ Some studies have also shown that CXCR4 and CXCR7 promote cell migration, invasion, angiogenesis, and tumorigenesis through different modes of signal transduction based on their respective characteristics. ${ }^{19,30,31}$ By establishing double-knockout cell lines of CXCR4 and CXCR7, we found that the co-knockout of CXCR4 and CXCR7 genes showed higher antitumor effects than the single knockout of CXCR4 or CXCR7 in vitro. Therefore, we suggest a new mechanism for breast cancer metastasis and support the theoretical basis of CXCR4/CXCR7 combined inhibition targeted therapy.

CRISPR/Cas9 is a gene editing technique developed in recent years that can efficiently and accurately knockout the genome; ${ }^{32}$ the CRISPR/Cas9 system consists of Cas9 protein and SgRNA, targeting a pair of SgRNAs to target the upstream and downstream of the target gene and recruiting Cas9 nuclease for cleavage, resulting in the use of nonhomologous end-joining mechanism for DNA repair, which is not accurate and can easily produce frame-shift mutations, to achieve the role as a knockout target gene. ${ }^{33}$ Compared with other gene-editing technologies such as zinc-finger nucleases and transcription activator-like effector nucleases, CRISPR/Cas9 system is easier, faster, and more accurate; compared with the RNA interference technique, the miss effect is completely controlled, and the target protein level is completely eliminated. The target range is not limited to transcripts but involves all genomic sequences, such as exons, introns, promoters, enhancers, and intergenic sequences. ${ }^{34}$ 
Chemokines and their receptors are very important to the progress of tumors. ${ }^{5}$ There are many reports on the effect of CXCR4 and CXCR7 gene silencing on tumor biological characteristics. For example, silencing the CXCR4 gene in the TNBC cell line can significantly inhibit the growth of tumor cells, reduce colony formation, and increase the sensitivity of cisplatin chemotherapy, ${ }^{9}$ and CXCR4 gene silencing can significantly block the metastasis of breast cancer. ${ }^{35}$ Additionally, the expression of the CXCR7 gene in breast cancer cells is silenced by RNA interference. The migration speed of breast cancer cells induced by CXCL12 was slowed down, and the invasion and adhesion of breast cancer cells were obviously weakened; ${ }^{16}$ however, there are no studies on the progression of TNBC after CXCR4 and CXCR7 single-target gene knockout and CXCR4 and CXCR7 target gene co-knockout.

In this study, we first used the CRISPR/Cas9 technique for single and co-knockout of the target genes of CXCR4 and CXCR7 in the TNBC cell line MDA-MB-231, and the biological effect of CXCR4 and CXCR7 target genes on the TNBC cell line. It was verified that CXCR4 and CXCR7 have synergistic effects after knockout. Several studies have shown that CXCR4 and CXCR7 promote the growth, proliferation, invasion, and distant metastasis of tumor cells in many malignant tumors, including breast and prostate cancers. . $^{19,36,37}$ Our results showed that, after the single knockout of the CXCR4 or CXCR7 target gene, the target protein is not expressed, significantly inhibiting the proliferation, growth, migration, and invasion of TNBC in vitro. At the same time, after we further knocked out the target gene of CXCR4 and CXCR7, the biological inhibition of TNBC was more significant; therefore, we speculate that there is a synergistic effect between CXCR4 and CXCR7 on the progression of TNBC. The chemokine receptor binds to its ligand CXCL12 and plays a corresponding role in promoting tumor progression. We conducted CXCL12 induction experiments during the migration and invasion assays. The results showed that both the control group and single knockout of the CXCR4 or CXCR7 target genome, the co-knockout of the CXCR4 and CXCR7 target genomes, and the addition of a certain concentration of CXCL12 significantly enhanced the migration and invasion functions. Therefore, the co-knockout of the CXCR4 and CXCR7 target genes is more effective in inhibiting the progression of TNBC and may provide a theoretical basis for the targeted treatment of TNBC. Through the results of this experiment, we can further study the specific signal pathways and mechanisms that affect TNBC after CXCR4 and CXCR7 knockout and the mechanism of inhibition of TNBC progress after co-knockout and single knockout, which may provide a new target for the treatment of TNBC.

\section{Conclusion}

In conclusion, the knockout of the CXCR4 or CXCR7 target gene can significantly inhibit the proliferation, growth, migration, and invasion of TNBC cells, and delay the progression of TNBC, but co-knockout of CXCR4 and CXCR7 target genes is more effective. The addition of CXCL12 enhanced the migration and invasion ability of TNBC, regardless of whether CXCR4 or CXCR7 was knocked out or not. These results suggest that $\mathrm{CXCL} 12, \mathrm{CXCR} 4$, and CXCR7 play an important part in the progression of TNBC, providing a potential target therapy for TNBC; however, its specific mechanism remains to be further investigated.

\section{Acknowledgments}

This work was supported by a grant from the Hunan Provincial Department of Science and Technology (No: 2018JJ2610).

\section{Disclosure}

The authors report no conflicts of interest in this work.

\section{References}

1. Ennour-Idrissi K, Maunsell E, Diorio C. Telomere length and breast cancer prognosis: a systematic review. Cancer Epidemiol Biomarkers Prev. 2017;26(1):3-10. doi:10.1158/1055-9965.EPI-16-0343

2. Veta M, Pluim JP, van Diest PJ, Viergever MA. Breast cancer histopathology image analysis: a review. IEEE Trans Biomed Eng. 2014; 61(5):1400-1411. doi:10.1109/TBME.2014.2303852

3. Rakha EA, El-Sayed ME, Green AR, Lee AH, Robertson JF, Ellis IO. Prognostic markers in triple-negative breast cancer. Cancer. 2007; 109(1):25-32. doi:10.1002/cncr.22381

4. Millikan RC, Newman B, Tse CK, et al. Epidemiology of basal-like breast cancer. Breast Cancer Res Treat. 2008;109(1):123-139. doi:10. 1007/s10549-007-9632-6

5. Sarvaiya PJ, Guo D, Ulasov I, Gabikian P, Lesniak MS. Chemokines in tumor progression and metastasis. Oncotarget. 2013;4(12):2171-2185. doi:10.18632/oncotarget.1426

6. Kang H, Watkins G, Parr C, Douglas-Jones A, Mansel RE, Jiang WG. Stromal cell derived factor-1: its influence on invasiveness and migration of breast cancer cells in vitro, and its association with prognosis and survival in human breast cancer. Breast Cancer Res. 2005;7(4): R402-R410. doi:10.1186/bcr1022

7. Lv ZD, Kong B, Liu XP, et al. CXCL12 chemokine expression suppresses human breast cancer growth and metastasis in vitro and in vivo. Int J Clin Exp Pathol. 2014;7(10):6671-6678.

8. Sun X, Cheng G, Hao M, et al. CXCL12/CXCR4/CXCR7 chemokine axis and cancer progression. Cancer Metastasis Rev. 2010;29(4): 709-722. doi:10.1007/s10555-010-9256-x

9. Teicher BA, Fricker SP. CXCL12 (SDF-1)/CXCR4 pathway in cancer. Clin Cancer Res. 2010;16(11):2927-2931. doi:10.1158/1078-0432. CCR-09-2329

10. Rubin JB. Chemokine signaling in cancer: one hump or two? Semin Cancer Biol. 2009;19(2):116-122. doi:10.1016/j.semcancer.2008. 10.001 
11. Palm D, Lang K, Brandt B, Zaenker KS, Entschladen F. In vitro and in vivo imaging of cell migration: two interdepending methods to unravel metastasis formation. Semin Cancer Biol. 2005;15(5):396-404. doi:10.1016/j.semcancer.2005.06.008

12. Muller A, Homey B, Soto H, et al. Involvement of chemokine receptors in breast cancer metastasis. Nature. 2001;410(6824):50-56. doi:10.1038/ 35065016

13. Park JM, Munoz JL, Won BW, et al. Exogenous CXCL12 activates protein kinase $\mathrm{C}$ to phosphorylate connexin 43 for gap junctional intercellular communication among confluent breast cancer cells. Cancer Lett. 2013;331(1):84-91. doi:10.1016/j.canlet.2012.12.007

14. Soon PS, Kim E, Pon CK, et al. Breast cancer-associated fibroblasts induce epithelial-to-mesenchymal transition in breast cancer cells. Endocr Relat Cancer. 2013;20(1):1-12. doi:10.1530/ERC-12-0227

15. Hinton CV, Avraham S, Avraham HK. Role of the CXCR4/CXCL12 signaling axis in breast cancer metastasis to the brain. Clin Exp Metastasis. 2010;27(2):97-105. doi:10.1007/s10585-008-9210-2

16. Miao Z, Luker KE, Summers BC, et al. CXCR7 (RDC1) promotes breast and lung tumor growth in vivo and is expressed on tumor-associated vasculature. Proc Natl Acad Sci U S A. 2007;104(40):15735-15740. doi:10.1073/pnas.0610444104

17. Wang J, Shiozawa Y, Wang J, et al. The role of CXCR7/RDC1 as a chemokine receptor for CXCL12/SDF-1 in prostate cancer. $J$ Biol Chem. 2008;283(7):4283-4294. doi:10.1074/jbc.M707465200

18. Zheng K, Li HY, Su XL, et al. Chemokine receptor CXCR7 regulates the invasion, angiogenesis and tumor growth of human hepatocellular carcinoma cells. J Exp Clin Cancer Res. 2010;29:31. doi:10.1186/17569966-29-17

19. Burns JM, Summers BC, Wang Y, et al. A novel chemokine receptor for SDF-1 and I-TAC involved in cell survival, cell adhesion, and tumor development. J Exp Med. 2006;203(9):2201-2213. doi:10.1084/ jem.20052144

20. Bauerle KT, Schweppe RE, Lund G, et al. Nuclear factor kappaBdependent regulation of angiogenesis, and metastasis in an in vivo model of thyroid cancer is associated with secreted interleukin-8. J Clin Endocrinol Metab. 2014;99(8):E1436-E1444. doi:10.1210/jc.2013-3636

21. Kollmar O, Rupertus K, Scheuer C, et al. CXCR4 and CXCR7 regulate angiogenesis and CT26.WT tumor growth independent from SDF-1. Int $J$ Cancer. 2010;126(6):1302-1315. doi:10.1002/ijc.24956

22. Hao M, Zheng J, Hou K, et al. Role of chemokine receptor CXCR7 in bladder cancer progression. Biochem Pharmacol. 2012;84(2):204-214. doi:10.1016/j.bcp.2012.04.007

23. Inaguma $\mathrm{S}$, Riku $\mathrm{M}$, Ito $\mathrm{H}$, Tsunoda $\mathrm{T}$, Ikeda $\mathrm{H}$, Kasai K. GLI1 orchestrates CXCR4/CXCR7 signaling to enhance migration and metastasis of breast cancer cells. Oncotarget. 2015;6(32):33648-33657. doi:10.18632/oncotarget.5203
24. Qin Y, Zhou Z, Zhang F, et al. Induction of regulatory B-cells by mesenchymal stem cells is affected by SDF-1alpha-CXCR7. Cell Physiol Biochem. 2015;37(1):117-130. doi:10.1159/000430338

25. Zhou SM, Zhang F, Chen XB, et al. miR-100 suppresses the proliferation and tumor growth of esophageal squamous cancer cells via targeting CXCR7. Oncol Rep. 2016;35(6):3453-3459. doi:10.3892/or.2016.4701

26. Anders CK, Carey LA. Biology, metastatic patterns, and treatment of patients with triple-negative breast cancer. Clin Breast Cancer. 2009; 9(Suppl 2):S73-S81. doi:10.3816/CBC.2009.s.008

27. Wu W, Qian L, Chen X, Ding B. Prognostic significance of CXCL12, CXCR4, and CXCR7 in patients with breast cancer. Int J Clin Exp Pathol. 2015;8(10):13217-13224.

28. Wu W, Qian L, Dai J, Ding B, Chen X. Expression of chemokine CXCL12 and its receptor (CXCR4 and CXCR7) in different molecular subtypes of human breast carcinoma and the clinical significance. Zhong Nan Da Xue Xue Bao Yi Xue Ban. 2017;42(2):147-153. doi:10.11817/j.issn.1672-7347.2017.02.005

29. Luker KE, Lewin SA, Mihalko LA, et al. Scavenging of CXCL12 by CXCR7 promotes tumor growth and metastasis of CXCR4-positive breast cancer cells. Oncogene. 2012;31(45):4750-4758. doi:10.1038/ onc. 2011.633

30. Hernandez L, Magalhaes MA, Coniglio SJ, Condeelis JS, Segall JE. Opposing roles of CXCR4 and CXCR7 in breast cancer metastasis. Breast Cancer Res. 2011;13(6):R128. doi:10.1186/bcr3052

31. Kerdivel G, Boudot A, Pakdel F. Estrogen represses CXCR7 gene expression by inhibiting the recruitment of NFkappaB transcription factor at the CXCR7 promoter in breast cancer cells. Biochem Biophys Res Commun. 2013;431(4):729-733. doi:10.1016/j.bbrc. 2013.01.050

32. Makarova KS, Haft DH, Barrangou R, et al. Evolution and classification of the CRISPR-Cas systems. Nat Rev Microbiol. 2011;9(6):467-477. doi: $10.1038 /$ nrmicro2577

33. Ran FA, Hsu PD, Lin CY, et al. Double nicking by RNA-guided CRISPR Cas9 for enhanced genome editing specificity. Cell. 2013;154(6): 1380-1389. doi:10.1016/j.cell.2013.08.021

34. Shalem O, Sanjana NE, Hartenian E, et al. Genome-scale CRISPR-Cas9 knockout screening in human cells. Science. 2014;343(6166):84-87. doi:10.1126/science. 1247005

35. Liang Z, Yoon Y, Votaw J, Goodman MM, Williams L, Shim H. Silencing of CXCR4 blocks breast cancer metastasis. Cancer Res. 2005;65(3): 967-971.

36. Gassmann P, Haier J, Schluter K, et al. CXCR4 regulates the early extravasation of metastatic tumor cells in vivo. Neoplasia. 2009;11(7): 651-661.

37. Mukherjee D, Zhao J. The role of chemokine receptor CXCR4 in breast cancer metastasis. Am J Cancer Res. 2013;3(1):46-57.
OncoTargets and Therapy

\section{Publish your work in this journal}

OncoTargets and Therapy is an international, peer-reviewed, open access journal focusing on the pathological basis of all cancers, potential targets for therapy and treatment protocols employed to improve the management of cancer patients. The journal also focuses on the impact of management programs and new therapeutic agents and protocols on
Dovepress

patient perspectives such as quality of life, adherence and satisfaction. The manuscript management system is completely online and includes a very quick and fair peer-review system, which is all easy to use. Visit http://www.dovepress.com/testimonials.php to read real quotes from published authors. 